\title{
Next Generation Fecal Microbiota Transplantation
}

\author{
Tae-Geun Gweon ${ }^{1,2}$ and Soo-Young Na,3 \\ ${ }^{1}$ Division of Gastroenterology, Department of Internal Medicine, College of Medicine, The Catholic University of Korea, Seoul, ${ }^{2}$ Division \\ of Gastroenterology, Department of Internal Medicine, Bucheon St. Mary's Hospital, College of Medicine, The Catholic University of \\ Korea, Bucheon, ${ }^{3}$ Division of Gastroenterology, Department of Internal Medicine, Incheon St. Mary's Hospital, College of Medicine, The \\ Catholic University of Korea, Incheon, Korea
}

Fecal microbiota transplantation (FMT) is considered as an effective treatment for Clostridioides difficile infection. However, the precise mechanism of FMT is yet to be determined. Human stool consists of the gut microbiota, bacterial debris, and metabolic products. Of these, the intestinal microbiota is the most important factor that exerts therapeutic efficacy in FMT. Fresh donor stool, blended with normal saline, has been employed for traditional FMT. Nevertheless, stool processing is a major impediment in FMT. Frozen stool and capsule formulations have similar efficacy to that of fresh stool. In addition, several novel stool products have been identified. A stool bank that provides stool products with pre-screened donor stool has been established to help physicians and thereby facilitate FMT. Recent next-generation sequencing techniques have been key in facilitating the detailed analysis of the microbiota and gut environment of individual donors and recipients. Clin Endosc 2021;54:152-156

Key Words: Clostridioides difficile infection; Fecal microbiota transplantation; Microbiota

\section{INTRODUCTION}

Fecal microbiota transplantation (FMT) is regarded as an effective treatment for Clostridioides difficile infection (CDI). ${ }^{1}$ For recurrent or refractory CDI, the treatment efficacy of FMT is as high as $90 \%$. With growing evidence of the relationship between dysbiosis and various diseases, including gastrointestinal and metabolic diseases, FMT has been investigated in various clinical settings other than CDI.

The process of FMT includes two major components: (1) the pre-FMT process, including donor screening and stool processing, to manufacture the fecal material, and (2) the de-

Received: January 16, 2021 Revised: February 23, 2021

Accepted: February 24, 2021

Correspondence: Soo-Young $\mathrm{Na}$

Division of Gastroenterology, Department of Internal Medicine, Incheon St.

Mary's Hospital, College of Medicine, The Catholic University of Korea, 56,

Dongsu-ro, Bupyeong-gu, Incheon 21431, Korea

Tel: +82-32-280-5052, Fax: +82-32-280-5987, E-mail: sktndud@hanmail.net

ORCID: https://orcid.org/0000-0003-3685-6823

It is the invited review article.

cc This is an Open Access article distributed under the terms of the Creative Commons Attribution Non-Commercial License (http://creativecommons.org/ licenses/by-nc/3.0) which permits unrestricted non-commercial use, distribution, and reproduction in any medium, provided the original work is properly cited. livery of stool products. Human stool consists of microorganisms, water, bacterial debris, DNA, and metabolic products, including short-chain fatty acids. Microorganisms in the gut consist of bacteria, viruses, fungi, and archaea. FMT has been developed to improve the gut microbial environment. In this article, we reviewed the development of stool processing, formulation, and application of precision medicine in FMT rather than clinical indications.

\section{FMT WITH CURRENTLY USED STOOL PRODUCTS}

\section{Fresh stool}

Traditionally, fresh stool of patient-directed donors, including families, relatives, or friends, was used for FMT. ${ }^{2,3}$ Once the stool was collected, it was homogenized with normal saline using a blender at a 1:3-1:5 ratio. The stool suspension was then filtered through a sterile gauze or stainless-steel sieve to remove the particles. The fresh fecal suspension was administered within 2 to $8 \mathrm{~h}$ of evacuation. ${ }^{2,3}$ However, the storage period can be extended at a lower temperature. An ex vivo study has suggested that fresh stool might be stored up to $24 \mathrm{~h}$ in a 
home refrigerator at $4^{\circ} \mathrm{C}{ }^{4}$ The means of FMT administration include nasogastric tube, enema, upper endoscopy, and colonoscopy. $^{3}$

\section{Frozen stool}

The use of frozen stool was first reported in a 2012 study, which demonstrated the screening and processing of donor stool. ${ }^{5}$ The 'ready to use' fecal suspension was stored at $-80^{\circ} \mathrm{C}$ in a refrigerator and thawed prior to FMT administration. The study had reported that the efficacy of FMT for the treatment of recurrent CDI, using frozen stool, was 90\% (19/21). Bacterial precipitants are essential for the long-term preservation of microorganisms. Glycerol at 10\% concentration is the most commonly used precipitant. A study has investigated the longterm viability of frozen stool. ${ }^{6}$ Two formulations were used in the study: (1) normal saline and $10 \%$ glycerol and (2) normal saline. The bacterial count was investigated post 2 and 6 months of storage. The results revealed that at two months, the bacterial counts of the two formulations were similar to those at the baseline. However, the bacterial count in the normal saline formulation significantly decreased at 6 months compared to that at the baseline. The bacterial count of the $10 \%$ glycerol formulation was similar to that of the baseline at 6 months. Further, the durability of frozen stool suspensions depends on the storage temperature. Frozen stool can be stored without depletion in viability, for 1 month at $-20^{\circ} \mathrm{C}$ and 6 to 12 months at $-80^{\circ} \mathrm{C} ., 6,7$

The treatment efficacy of frozen stool is reportedly comparable to that of fresh stool. ${ }^{5,7}$ A randomized controlled trial (RCT) showed that the efficacy of frozen stool is comparable to that of fresh stool in FMT administered via enema (frozen, 76/91 [83.5\%] vs fresh, 74/87 [85.1\%]). ${ }^{7}$ Additionally, frozen stool has several advantages over fresh stool. ${ }^{5}$ Foremost, the foul odor of stool suspension is amended in frozen stool as compared to the fresh stool. Second, the FMT can be performed with a reduced latency time with frozen stool. The development of frozen stool has facilitated the establishment of a non-profit stool bank.

\section{Stool bank}

The dirty aspect of stool preparation is a critical barrier to FMT. Furthermore, it is challenging to process the stool in a hospital owing to hygiene considerations. Here, the service of a stool bank can eliminate the time and effort required to screen and process stool material for physicians. As the procedure for stool processing is standardized, the volume of feces and fecal suspension provided by the stool bank is reliable. Techniques for manufacturing frozen stool are essential to run a stool bank. ${ }^{8}$ Healthy, unrelated donors (known as universal donors) voluntarily donate their stool compared to patient-directed donors, including families, relatives, or friends. Volunteers of stool donation are screened through interviews, blood tests, and stool tests. ${ }^{8}$ The donors are also regularly screened for their suitability to perform stool donation. Furthermore, the universal donors can donate their stools repeatedly within 2-3 months that enables reduction in the cost of screening. In addition, the lead time to conduct FMT could be considerably reduced using FMT products provided by the stool bank as compared to that required in stool processing by a physician. The stool product of donors, provided for FMT, should be traceable in terms of success for FMT and adverse events. ${ }^{8}$ Openbiome (Cambridge, MA, USA) is the first non-profit stool bank in the world, which was founded in 2012. In Korea, two non-profit stool banks (Bioeleven, Seoul; Microbiotics, Seoul, Korea) have been established till date.

\section{FMT WITH NEXT GENERATION STOOL FORMULATIONS}

\section{Liquid capsule}

Conventional FMT infuses fresh or frozen liquid fecal suspensions (200 to $500 \mathrm{~mL}$ ) to the recipients. Frequently, FMT has been attempted through endoscopic infusion. Large amounts of liquid infusion, especially through upper endoscopy, have a risk of aspiration, which is often fatal. ${ }^{10}$ Youngster et al. have reported the use of frozen capsules. ${ }^{11}$ Production of the capsules involved the concentration of fecal suspension by centrifugation and the volume reduction to $1 / 10$ of the initial volume. The final volume of the capsule was $0.65 \mathrm{~mL}$ and one capsule contained $1.6 \mathrm{~g}$ of stool. The patients were subjected to ingestion of 30 capsules during two consecutive days, which was comparable to ingestion of $48 \mathrm{~g}$ of stool. The success rate of FMT for the treatment of CDI using the frozen capsules was found to be $90 \%$. An RCT reported that FMT using the capsules was not inferior to that using colonoscopic infusion in patients with recurrent CDI (capsule, 96.2\% [51/53] vs colonoscopic infusion, $96.2 \%$ [50/52]; risk difference, $0 \%$ ). ${ }^{12}$ Further, the capsule- mediated FMT was used for eradication of Carbapenemase-producing Enterobacteriaceae (CPE) colonization. ${ }^{13} \mathrm{~A}$ total of 30 oral capsules, containing 25 to $30 \mathrm{~g}$ stool in total, were ingested during two consecutive days. The results revealed that of the 13 patients who finished the course of 30 capsules, CPE was eradicated in $9(69.2 \%, 9 / 13)$ one month after the FMT. The conditions for storage of liquid capsules, including the duration and temperature, are similar to those of frozen stool. ${ }^{6,711}$ Additionally, FMT using capsules can be performed by the patients themselves without the help of 
physicians. However, capsule FMT is not available for patients with swallowing difficulties.

\section{Lyophilized capsule}

Feces can be further concentrated and dehydrated to make a powdered formulation for preservation. The lyophilization process can concentrate the volume of the final product. The final lyophilized product weighs 1.5 to $4.9 \mathrm{~g}$ from $100 \mathrm{~g}$ of donated stool. ${ }^{14,15}$ Lyophilized capsules can be stored at a higher temperature of $4^{\circ} \mathrm{C}$ for up to 12 months. ${ }^{16}$ In a RCT that compared the efficacy of fresh, frozen, and lyophilized formulations for CDI, the FMT was performed with colonoscopy in three groups. ${ }^{17}$ The lyophilized stool product was mixed with $250 \mathrm{~mL}$ of normal saline and infused using colonoscopy. The cure rates in the fresh, frozen, and lyophilized formulations were $100 \%$ (25/20), 83\% (20/24), and 78\% (16/23), respectively. Evidently, the treatment efficacy of the lyophilized group was lower than that of the fresh group $(p=0.022)$. Bacterial diversity of FMT recipients assessed post 7 and 14 days of FMT was found to be lowest in the lyophilized group among the three groups. However, cryoprotectants were not supplemented when fecal powder was produced in this study. Furthermore, re-FMT was not attempted in patients who initially demonstrated FMT failure. The same research group supplemented cryoprotectants during the manufacturing of lyophilized capsules, in another RCT that compared lyophilized capsules and enema using frozen stool for the treatment of CDI. ${ }^{14}$ The results of the trial revealed that after 2 months of FMT, CDI did not recur in $84 \%$ of the patients administered lyophilized capsules and $88 \%$ of those administered enema $(p=0.73)$. In summary, the efficacy of lyophilized FMT for the treatment of CDI is $78 \%-87.5 \%$, which is comparable to that of FMT using frozen or fresh stool. ${ }^{14,15,17}$ However, the amount of stool used for lyophilized FMT differed between the studies (50-200 g).

\section{Specific bacterial product}

Pathogen transmission is one of the most important adverse events associated with FMT. Lately, antibiotic-resistant bacterial transmission has been reported, which has resulted in the death of FMT recipients. ${ }^{18}$ Rigorous screening cannot perfectly eliminate the possibility of pathogen transmission. In this regard, techniques for eliminating pathogens and isolating beneficial microorganisms have gained interest. Petrof et al. isolated 33 commensal bacterial species that are sensitive to antimicrobials. ${ }^{19}$ Each of the isolated microbes was cultured on anaerobic agar individually. A mixture of bacterial suspension, named RePOOPulate, was used as a stool substitute formulation (synthetic stool). Two patients with recurrent CDI were cured with colonoscopic infusion of RePOOPulate.

\section{Sterile fecal filtrate}

The exact mechanism of FMT has not yet been completely elucidated. Although microbiota is the most important component of human stool, the remaining components of human stool, such as the bacterial debris or short chain fatty acids, might also exert therapeutic efficacy in FMT. Ott et al. hypothesized that stool components, other than living microorganisms, have clinical efficacy for CDI therapy. ${ }^{20}$ In this study, the fecal suspension was centrifuged post homogenization and blending. The supernatant was filtered through a fine paper filter (5 to $10 \mu \mathrm{m}$ pore size) to remove living microorganisms. The sterile filtrate was then transferred to the recipient using a nasojejunal tube. The results revealed that all the five patients with CDI recovered after FMT. Thus, transferring sterile fecal filtrate can be a good alternative to traditional FMT, especially for immunocompromised patients, to reduce the risk of pathogen transmission. Despite this impressive study, till date, sterile fecal material has not been investigated for non-CDI diseases.

\section{Anaerobic processing}

Most gut bacteria are anaerobes. Consequently, stool processing under aerobic conditions results in the loss of bacterial diversity and reduced biosynthesis of butyrate, a short-chain fatty acid that is essential for homeostasis. ${ }^{21}$ In addition, the viability of bacteria is lower in aerobic processing than in frozen-thawed suspension in anaerobic processing. For patients with ulcerative colitis (UC), FMT employing anaerobically processed stool resulted in a higher remission rate as compared to that with an autologous FMT, wherein aerobically processed stool was employed $(p=0.03) .{ }^{22}$ However, the remission rate in the anaerobically processed FMT was $32 \%(12 / 38)$, which was comparable to that in previous studies wherein stool was processed under aerobic conditions. ${ }^{23}$ Because of the high efficacy of aerobically processed FMT in CDI, anaerobic processing of stools for FMT has not been attempted for CDI. As a result, anaerobic processing is not mandatory for $\mathrm{CDI}$ in the current guidelines. ${ }^{24}$ However, the efficacy of anaerobic processing for FMT should be investigated in future studies, especially for non-CDI diseases such as metabolic disorders and inflammatory bowel disease.

\section{Spore formulation}

The spores of intestinal microbiota have been investigated for CDI and UC. ${ }^{25-27}$ In order to prepare spore formulation, the centrifuged fecal suspensions are mixed with ethanol to eradicate live gram-negative bacteria. The spore formulation 
is then converted into capsules. Once ingested, the spores of the donor's microbiota get engrafted and replicate in the recipient. Engrafted microbiota can suppress CDI recurrence and improve dysbiosis. Furthermore, the spores can be manufactured from a single strain (non-toxigenic Clostridioides difficile M3 strain) or a combination of the Firmicutes phylum. ${ }^{25,26,27}$ The process safety is a major advantage of spore formulation because the purification process of spores selectively eliminates vegetative bacteria, fungi, and viruses. Although spore formulation results in favorable primary efficacy for CDI and UC, the dosage and duration of ingestion of spore formulation should be tested in future studies.

\section{PRECISION MEDICINE}

Treatment to restore and modulate the human intestinal microbial environment includes FMT, probiotics, and prebiotics. Recent next-generation sequencing techniques can be used to analyze the microbiota at the species and strain levels, which is known to have functional and clinical relevance. ${ }^{28}$ Moreover, accumulating evidence suggests that dysbiosis can be corrected by FMT. ${ }^{28,29}$

Studies have shown that the diversity of the intestinal microbiota of FMT recipients increases post FMT. In addition to diversity, FMT can also alter the microbial composition. Changes in specific microbial taxa have been suggested as important factors for the efficacy of FMT, especially for non-CDI diseases. For instance, Bifidobacterium bifidum abundance has been reported to increase after FMT for CPE.$^{13}$ However, the bacterial composition after FMT differed between those who showed remission (FMT responders) and lack of remission (non-responders) in UC patients. ${ }^{29}$ Compared to non-responders, Eubacterium halli, Roseburia inulinivorans, and Ruminococcus bromii have been reported to be enriched in patients who show remission after FMT. A recent study proposed the term 'super-donor' that is defined as a donor whose stool shows favorable clinical efficacy in $\mathrm{FMT}^{30}$ The possibility of a super-donor may provide insights into donor-recipient interaction and specified microbiome identification.

\section{CONCLUSIONS}

The development of various stool formulations could enhance physician convenience, thereby eliminating obstacles to FMT. Traditional FMT is conceptually the transfer of whole microbiota of the donor to the patient. Studies on predefined, selected microbiota transfer for specific diseases are currently in progress. Efforts have been devoted to identify the factors contributing to the success of FMT, especially in non-CDI diseases, to enhance the success rate of FMT in these diseases. Thus, individualized therapy of patients that employs (1) matched donor selection or (2) defined microbial consortia, holds great potential for management of not only CDI but also non-CDI-related diseases in the future.

Conflicts of Interest

The authors have no potential conflicts of interest.

Funding

None.

Acknowledgement

We thank Ji Eun Bang, Kyunghye Jang, Ae Jin Kim, and Young Eun An for their outstanding assistance and help.

ORCID

Tae-Geun Gweon:

Soo-Young Na:

https://orcid.org/0000-0002-0884-7228

https://orcid.org/0000-0003-3685-6823

\section{REFERENCES}

1. Quraishi MN, Widlak M, Bhala N, et al. Systematic review with meta-analysis: the efficacy of faecal microbiota transplantation for the treatment of recurrent and refractory Clostridium difficile infection. Aliment Pharmacol Ther 2017;46:479-493.

2. Brandt LJ, Aroniadis OC, Mellow M, et al. Long-term follow-up of colonoscopic fecal microbiota transplant for recurrent Clostridium difficile infection. Am J Gastroenterol 2012;107:1079-1087.

3. Kim KO, Gluck M. Fecal microbiota transplantation: an update on clinical practice. Clin Endosc 2019;52:137-143.

4. Burz SD, Abraham AL, Fonseca F, et al. A guide for Ex Vivo handling and storage of stool samples intended for fecal microbiota transplantation. Sci Rep 2019;9:8897.

5. Hamilton MJ, Weingarden AR, Sadowsky MJ, Khoruts A. Standardized frozen preparation for transplantation of fecal microbiota for recurrent Clostridium difficile infection. Am J Gastroenterol 2012;107:761-767.

6. Costello SP, Conlon MA, Vuaran MS, Roberts-Thomson IC, Andrews JM. Faecal microbiota transplant for recurrent Clostridium difficile infection using long-term frozen stool is effective: clinical efficacy and bacterial viability data. Aliment Pharmacol Ther 2015;42:1011-1018.

7. Lee CH, Steiner T, Petrof EO, et al. Frozen vs Fresh fecal microbiota transplantation and clinical resolution of diarrhea in patients with recurrent Clostridium difficile infection: a randomized clinical trial. JAMA 2016;315:142-149.

8. Terveer EM, van Beurden YH, Goorhuis A, et al. How to: establish and run a stool bank. Clin Microbiol Infect 2017;23:924-930.

9. Kim KO, Schwartz MA, Lin OST, Chiorean MV, Gluck M. Reducing cost and complexity of fecal microbiota transplantation using universal donors for recurrent Clostridium difficile infection. Adv Ther 2019;36:2052-2061.

10. Wang S, Xu M, Wang W, et al. Systematic review: adverse events of fecal microbiota transplantation. PLoS One 2016;11:e0161174.

11. Youngster I, Russell GH, Pindar C, Ziv-Baran T, Sauk J, Hohmann EL. Oral, capsulized, frozen fecal microbiota transplantation for relapsing 
Clostridium difficile infection. JAMA 2014;312:1772-8.

12. Kao D, Roach B, Silva M, et al. Effect of oral capsule- vs colonoscopy-delivered fecal microbiota transplantation on recurrent clostridium difficile infection: a randomized clinical trial. JAMA 2017;318:1985-1993.

13. Bar-Yoseph H, Carasso S, Shklar S, et al. Oral capsulized fecal microbiota transplantation for eradication of carbapenemase-producing Enterobacteriaceae colonization with a metagenomic perspective. Clin Infect Dis 2020.

14. Jiang ZD, Jenq RR, Ajami NJ, et al. Safety and preliminary efficacy of orally administered lyophilized fecal microbiota product compared with frozen product given by enema for recurrent Clostridium difficile infection: a randomized clinical trial. PLoS One 2018;13:e205064.

15. Reigadas E, Bouza E, Olmedo M, et al. Faecal microbiota transplantation for recurrent Clostridioides difficile infection: experience with lyophilized oral capsules. J Hosp Infect 2020;105:319-324.

16. Reygner J, Charrueau C, Delannoy J, et al. Freeze-dried fecal samples are biologically active after long-lasting storage and suited to fecal microbiota transplantation in a preclinical murine model of Clostridioides difficile infection. Gut Microbes 2020;11:1405-1422.

17. Jiang ZD, Ajami NJ, Petrosino JF, et al. Randomised clinical trial: faecal microbiota transplantation for recurrent Clostridum difficile infection fresh, or frozen, or lyophilised microbiota from a small pool of healthy donors delivered by colonoscopy. Aliment Pharmacol Ther 2017;45:899908.

18. DeFilipp Z, Bloom PP, Torres Soto M, et al. Drug-resistant E. coli bacteremia transmitted by fecal microbiota transplant. N Engl J Med 2019;381:2043-2050.

19. Petrof EO, Gloor GB, Vanner SJ, et al. Stool substitute transplant therapy for the eradication of Clostridium difficile infection: 'RePOOPulating' the gut. Microbiome 2013;1:3.

20. Ott SJ, Waetzig GH, Rehman A, et al. Efficacy of sterile fecal filtrate transfer for treating patients with Clostridium difficile infection. Gastro- enterology 2017;152:799-811.e7.

21. Papanicolas LE, Choo JM, Wang Y, et al. Bacterial viability in faecal transplants: which bacteria survive? EBioMedicine 2019;41:509-516.

22. Costello SP, Hughes PA, Waters O, et al. Effect of fecal microbiota transplantation on 8-week remission in patients with ulcerative colitis: a randomized clinical trial. JAMA 2019;321:156-164.

23. Imdad A, Nicholson MR, Tanner-Smith EE, et al. Fecal transplantation for treatment of inflammatory bowel disease. Cochrane Database Syst Rev 2018;11:Cd012774.

24. Mullish BH, Quraishi MN, Segal JP, et al. The use of faecal microbiota transplant as treatment for recurrent or refractory Clostridium difficile infection and other potential indications: joint British Society of Gastroenterology (BSG) and Healthcare Infection Society (HIS) guidelines. Gut 2018;67:1920-1941.

25. Gerding DN, Meyer T, Lee C, et al. Administration of spores of nontoxigenic Clostridium difficile strain M3 for prevention of recurrent C. difficile infection: a randomized clinical trial. JAMA 2015;313:1719-1727.

26. Khanna S, Pardi DS, Kelly CR, et al. A Novel Microbiome therapeutic increases gut microbial diversity and prevents recurrent Clostridium difficile infection. J Infect Dis 2016;214:173-181.

27. Henn MR, O'Brien EJ, Diao L, et al. A phase $1 b$ safety study of SER287, a spore-based microbiome therapeutic, for active mild to moderate ulcerative colitis. Gastroenterology 2021;160:115-127.e30.

28. Li SS, Zhu A, Benes V, et al. Durable coexistence of donor and recipient strains after fecal microbiota transplantation. Science 2016;352:586-589.

29. Paramsothy S, Nielsen S, Kamm MA, et al. Specific bacteria and metabolites associated with response to fecal microbiota transplantation in patients with ulcerative colitis. Gastroenterology 2019;156:1440-1454.e2.

30. Moayyedi P, Surette MG, Kim PT, et al. Fecal microbiota transplantation induces remission in patients with active ulcerative colitis in a randomized controlled trial. Gastroenterology 2015;149:102-109.e6. 